УДК 517.958

doi:10.13108/2017-9-1-41

UDC 517.958

\title{
MODELLING COMPRESSION WAVES WITH \\ A LARGE INITIAL GRADIENT IN THE KORTEWEG-DE VRIES HYDRODYNAMICS
}

\author{
S.V. ZAKHAROV, A.E. ELBERT
}

\begin{abstract}
We consider the Cauchy problem for the Korteweg-de Vries equation with a small parameter at the higher derivative and a large gradient of the initial function. By means of the numerical and analytic methods we show that the formal asymptotics obtained by renormalization is an asymptotic solution to the KdV equation. We obtain the graphs of the asymptotic solutions including the case of non-monotone initial data.
\end{abstract}

Keywords: Korteweg-de Vries equation, Cauchy problem, compression wave

Mathematics Subject Classification: 35Q53

\section{Formulation of the PROBlem}

We consider the Cauchy problem for the Korteweg-de Vries equation

$$
\begin{array}{ll}
\frac{\partial u}{\partial t}+u \frac{\partial u}{\partial x}+\varepsilon \frac{\partial^{3} u}{\partial x^{3}}=0, & t \geqslant 0, \quad \varepsilon>0, \\
u(x, 0, \varepsilon, \rho)=\Lambda\left(\frac{x}{\rho}\right), & x \in \mathbb{R}, \quad \rho>0,
\end{array}
$$

with a bounded initial function $\Lambda$ having finite limits $\Lambda_{0}^{ \pm}=\lim _{s \rightarrow \pm \infty} \Lambda(s), \Lambda_{0}^{+}<\Lambda_{0}^{-}$, whose derivative sufficiently fast tends to zero at infinity. This is a classical model of nonlinear waves propagation in a media with a small dispersion. For a discontinuous initial function, A.V. Gurevich and L.P. Pitaevskii studied the asymptotics [1]. The asymptotic formulae for the evolution of the compression waves were also found in works [2], [3] by the Whitham method and in work [4 by the inverse scattering problem method. For the step-like initial data the asymptotic formulae were obtained by the inverse scattering problem method [5]-[7]. In the case of a smoothed step the asymptotic expansions were constructed by the matching method in work [8].

We assume that the initial function $\Lambda: \mathbb{R} \rightarrow \mathbb{R}$ has finite limits $\Lambda_{0}^{ \pm}=\lim _{\sigma \rightarrow \pm \infty} \Lambda(\sigma)$ and the asymptotic expansions

$$
\Lambda(\sigma)=\sum_{n=0}^{\infty} \frac{\Lambda_{n}^{ \pm}}{\sigma^{n}}, \quad \sigma \rightarrow \pm \infty,
$$

S.V. Zakharov, A.E. Elbert, Modelling compression waves with a large initial gradient in THE KorteWEG-DE VRIES hydrodynamics.

(c) Zakharov S.V., Elbert A.E. 2017.

The work is supported by the complex program of fundamental scientific researches of UB RAS (project "Developing of new analytic, numeric and asymptotic methods for studying problems of mathematical physics and their applications to signal processing".

Submitted December 04, 2015. 
hold true. The existence of the classic smooth solution of the problem (1)-(2) is ensured by Kappeler theorem [9] if

$$
\int_{-\infty}^{0}\left|\Lambda(x)-\Lambda_{0}^{-}\right|\left(1-x^{3}\right) d x<\infty, \quad \int_{0}^{\infty}\left|\Lambda(x)-\Lambda_{0}^{+}\right|\left(1+x^{3}\right) d x<\infty .
$$

However, in the present work we can omit these conditions and consider only a formal asymptotic solution. Moreover, the existence of the solution with unbounded initial data was proved in a special class of functions [10].

In the present work we show that the approximation constructed in work [11] is an asymptotic solution to problem (1)-(2) as $\varepsilon \rightarrow 0, \rho \rightarrow 0$ and $\rho^{2} / \varepsilon \rightarrow 0$, and we also make a numerical analysis of this solution. The aim of present paper is to understand and define the precise mathematical meaning of formal "solutions" to the KdV equation. In particular, in the studying there arises a question in which sense one should treat the Gurevich-Pitaevskii solution [1] since the authors failed to find a precise mathematical formulation both in their original work and in works by specialists. In what follows, in Theorem 1 in Section 3 we provide a rigorous definition of the asymptotic solution inside the oscillation zone. Since in a similar problem for a parabolic equations one of the authors succeeded to show that the asymptotics obtained by the renormalization method is close to the exact solution [15], this is a reason to conjecture that a formal asymptotic solution found for the KdV equation by the same method also approximates an exact solution.

It is clear that the structure of the asymptotics should depend essentially on the relations between the parameters $\varepsilon$ and $\rho$. Here we assume the following condition:

$$
\mu=\frac{\rho}{\sqrt{\varepsilon}} \rightarrow 0
$$

\section{AsYMPTOTIC SOLUTION}

It is known that in a series of cases, the behavior of the solutions to singularly perturbed differential equations with a small parameter at a higher derivatives is in some sense selfsimilar. Then an effective approach to analyse the solution is by means of renorm-group [12]. This approach has an advantage that it allows to get an uniform approximation of the problem and one does not have to construct asymptotic ansatzes in different domains. For instance, the compound asymptotics for the solution of the Cauchy problem with condition (2) was obtained for a quasilinear parabolic equation [14] by the matching method [13] and it was shown in work [15] that the renormalized approximation of the solution is asymptotically close to the compound asymptotic solution.

Let us construct an asymptotic solution of problem (1)-(2) by employing a technique similar to the renorm-group method in its simplest version. We pass to the inner variables

$$
x=\sqrt{\varepsilon} \eta, \quad t=\sqrt{\varepsilon} \theta
$$

since it allows us to take into consideration all terms of equation (1). As the first term we take the solutions to the equation

with the initial condition

$$
\frac{\partial Z}{\partial \theta}+Z \frac{\partial Z}{\partial \eta}+\frac{\partial^{3} Z}{\partial \eta^{3}}=0
$$

$$
Z(\eta, 0)= \begin{cases}\Lambda_{0}^{-}, & \eta<0 \\ \Lambda_{0}^{+}, & \eta>0\end{cases}
$$

We seek the expansion of the solution as

$$
u(x, t, \varepsilon, \rho)=Z(\eta, \theta)+\mu W(\eta, \theta, \mu)+\ldots,
$$


where the additional term $\mu W(\eta, \theta, \mu)$ is to remove the singularity of the first term at the initial time. Equations (1) and (5) yield that the function $W$ satisfies the linear equation

$$
\frac{\partial W}{\partial \theta}+\frac{\partial(Z W)}{\partial \eta}+\frac{\partial^{3} W}{\partial \eta^{3}}=0 .
$$

Differentiating equation (5) w.r.t. the variable $\eta$, we confirm that the expression

$$
G(\eta, \theta)=\frac{1}{\Lambda_{0}^{+}-\Lambda_{0}^{-}} \frac{\partial Z(\eta, \theta)}{\partial \eta}
$$

satisfies equation (8). Moreover, $G$ is the Green function since

$$
\lim _{\theta \rightarrow+0} \int_{-\infty}^{\infty} G(\eta, \theta) f(\eta) d \eta=-\frac{1}{\Lambda_{0}^{+}-\Lambda_{0}^{-}} \int_{-\infty}^{\infty} Z(\eta, 0) f^{\prime}(\eta) d \eta=f(0)
$$

for each compactly supported function $f$.

We choose the solution $W$ as the convolution with the Green function $G$ so that the asymptotic approximation satisfies initial condition (2). Then

$$
W=\frac{1}{\Lambda_{0}^{+}-\Lambda_{0}^{-}} \int_{-\infty}^{\infty} \frac{\partial Z(\eta-\mu s, \theta)}{\partial \eta}[\Lambda(s)-Z(s, 0)] d s .
$$

Integrating by parts and substituting into expansion (7), we obtain the sought expression

$$
u(x, t, \varepsilon, \rho) \approx \frac{1}{\Lambda_{0}^{+}-\Lambda_{0}^{-}} \int_{-\infty}^{\infty} Z\left(\frac{x-\rho s}{\sqrt{\varepsilon}}, \frac{t}{\sqrt{\varepsilon}}\right) \Lambda^{\prime}(s) d s,
$$

which clarifies the structure of the asymptotic solution w.r.t. the parameters $\varepsilon$ and $\rho$ at the main approximation. Despite the functions $Z$ and $W$ are independent explicitly on $\sqrt{\varepsilon}$, the result depends on $\mu$ and $\sqrt{\varepsilon}$ by change (4) since asymptotic solution (9) is considered also for finite values of the time $t$.

As an example we let $\Lambda_{0}^{+}=0$ and $\Lambda_{0}^{-}=a>0$. In the right hand side in $(9)$ we replace $Z(\eta, \theta)$ by the Gurevich-Pitaevskii asymptotic solution:

$$
\begin{aligned}
& Z_{\mathrm{GP}}(\eta, \theta)=a \quad \text { as } \quad \eta<-a \theta, \quad Z_{\mathrm{GP}}(\eta, \theta)=0 \quad \text { as } \quad \eta>2 a \theta / 3, \\
& Z_{\mathrm{GP}}(\eta, \theta)=a\left[2 \operatorname{dn}^{2}\left(\frac{a^{3 / 2} \theta}{\sqrt{6}}\left(\zeta-\frac{1+\sigma^{2}(\zeta)}{3}\right), \sigma(\zeta)\right)-1+\sigma^{2}(\zeta)\right] \quad \text { as } \quad-a \theta \leqslant \eta \leqslant 2 a \theta / 3
\end{aligned}
$$

where $\zeta=\frac{\eta}{a \theta}, \operatorname{dn}(v, \sigma)$ is the elliptic Jacobi function, and the function $\sigma(\zeta)$ is determined by the identity

$$
1+\sigma^{2}-\frac{2 \sigma^{2}\left(1-\sigma^{2}\right) K(\sigma)}{E(\sigma)-\left(1-\sigma^{2}\right) K(\sigma)}=3 \zeta
$$

$K(\sigma)$ and $E(\sigma)$ are the complete elliptic integral of the first and second kind. As a result we obtain

$$
\begin{aligned}
u(x, t, \varepsilon, \rho) \approx 2 & \Lambda\left(\frac{x+a t}{\rho}\right)-\Lambda\left(\frac{x-2 a t / 3}{\rho}\right) \\
& -\int_{(x-2 a t / 3) / \rho}^{(x+a t) / \rho} \Lambda^{\prime}(s)\left[2 \operatorname{dn}^{2}\left(\sqrt{\frac{a}{6 \varepsilon}} q(x, t, s, \rho), \sigma\left(\frac{x-\rho s}{a t}\right)\right)+\sigma^{2}\left(\frac{x-\rho s}{a t}\right)\right] d s,
\end{aligned}
$$


where

$$
q(x, t, s, \rho)=x-\rho s-\frac{a t}{3}\left[1+\sigma^{2}\left(\frac{x-\rho s}{a t}\right)\right] .
$$

By the change $x-\rho s=a t y$ we arrive at the following formula:

$$
\begin{aligned}
u(x, t, \varepsilon, \rho) \approx & u_{0}(x, t, \varepsilon, \rho)=2 \Lambda\left(\frac{x+a t}{\rho}\right)-\Lambda\left(\frac{x-2 a t / 3}{\rho}\right) \\
& -\frac{a t}{\rho} \int_{-1}^{2 / 3} \Lambda^{\prime}\left(\frac{x-a t y}{\rho}\right)\left[2 \operatorname{dn}^{2}\left(\frac{a^{3 / 2} t \omega(y)}{\sqrt{\varepsilon}}, \sigma(y)\right)+\sigma^{2}(y)\right] d y,
\end{aligned}
$$

where

$$
\omega(y)=\frac{1}{\sqrt{6}}\left\{y-\frac{1}{3}\left[1+\sigma^{2}(y)\right]\right\} .
$$

Thus, in this case the main asymptotic approximation is a function of three variables $x \rho^{-1}$, $t \rho^{-1}$ and $t \varepsilon^{-1 / 2}$, which coincides with the initial function $\Lambda\left(x \rho^{-1}\right)$ as $t=0$.

3. Analytic estimate of the value of KDV OPERATOR INSide the Oscillation ZONE

\section{Lemma 1. Let}

$$
\Lambda(s)=a+O\left(|s|^{-1}\right), \quad s \rightarrow-\infty, \quad \Lambda(s)=O\left(s^{-1}\right), \quad s \rightarrow+\infty,
$$

and these relations can be differentiated as many times as we need. Then for a bounded function $\varphi(z) \in C^{\infty}$ we have

$$
\frac{1}{\rho} \int_{-\infty}^{\infty} \Lambda^{\prime}\left(\frac{z}{\rho}\right) \varphi(z) d z=-a \varphi(0)+O(\sqrt{\rho}), \quad \rho \rightarrow 0 .
$$

Proof. We employ the fact that $\varphi(z)=\varphi(0)+O(\sqrt{\rho})$ as $-\sqrt{\rho} \leqslant z \leqslant \sqrt{\rho}$. We have

$$
\begin{aligned}
\frac{1}{\rho} \int_{-\infty}^{\infty} \Lambda^{\prime}\left(\frac{z}{\rho}\right) \varphi(z) d z+a \varphi(0)= & \frac{1}{\rho} \int_{-\sqrt{\rho}}^{\sqrt{\rho}} \Lambda^{\prime}\left(\frac{z}{\rho}\right) \varphi(z) d z+a \varphi(0) \\
& +\frac{1}{\rho} \int_{\sqrt{\rho}}^{\infty} \Lambda^{\prime}\left(\frac{z}{\rho}\right) \varphi(z) d z+\frac{1}{\rho} \int_{-\infty}^{-\sqrt{\rho}} \Lambda^{\prime}\left(\frac{z}{\rho}\right) \varphi(z) d z \\
= & \varphi(0)\left[\frac{1}{\rho} \int_{-\sqrt{\rho}}^{\sqrt{\rho}} \Lambda^{\prime}\left(\frac{z}{\rho}\right) d z+a\right]+O(\sqrt{\rho})=O(\sqrt{\rho})
\end{aligned}
$$

where we have employed the asymptotics for $\Lambda$ and $\Lambda^{\prime}$ as $s \rightarrow \pm \infty$. The proof is complete.

Corollary 1. The identities

$$
\begin{array}{ll}
\left(\frac{a t}{\rho}\right) \int_{-1}^{2 / 3} \Lambda^{\prime}\left(\frac{x-a t y}{\rho}\right) \varphi(y) d y=-a \varphi\left(\frac{x}{a t}\right)+O(\sqrt{\rho}), & \rho \rightarrow 0 \\
\left(\frac{a t}{\rho}\right)^{2} \int_{-1}^{2 / 3} \Lambda^{\prime \prime}\left(\frac{x-a t y}{\rho}\right) \varphi(y) d y=-a \varphi^{\prime}\left(\frac{x}{a t}\right)+O(\sqrt{\rho}+\rho / \varepsilon), \quad \rho \rightarrow 0
\end{array}
$$


hold true.

Theorem 1. For each function $\Lambda$ satisfying the assumptions of Lemma 1, the function $u_{0}$ defined by formula (11) is an asymptotic solution of equation (1) in the domain

$$
D_{0}=\left\{(x, t) \mid-a t+\sqrt{\varepsilon} \leqslant x \leqslant \frac{2 a t}{3}-\sqrt{\varepsilon}\right\}
$$

in the following sense:

$$
\begin{aligned}
\left|\mathscr{L} u_{0}\right| & \leqslant \frac{M}{\sqrt{\varepsilon}}\left(\frac{\rho}{\sqrt{\varepsilon}}+\sqrt{\rho \varepsilon}\right), \quad \frac{m(x, t, \varepsilon)}{\sqrt{\varepsilon}} \leqslant\left|\frac{\partial u_{0}}{\partial t}\right|+\left|u_{0} \frac{\partial u_{0}}{\partial x}\right|+\varepsilon\left|\frac{\partial^{3} u_{0}}{\partial x^{3}}\right| \leqslant \frac{M}{\sqrt{\varepsilon}}, \\
\text { as } \frac{\rho}{\sqrt{\varepsilon}}=\mu \rightarrow 0, \varepsilon \rightarrow 0, \text { where } & \\
\mathscr{L} u_{0} & =\frac{\partial u_{0}}{\partial t}+u_{0} \frac{\partial u_{0}}{\partial x}+\varepsilon \frac{\partial^{3} u_{0}}{\partial x^{3}}, \quad m(x, t, \varepsilon)=\frac{4 \sqrt{6} a^{5 / 2} \sigma^{2}}{3} \operatorname{dn}^{3}(b, \sigma) \operatorname{cn}(b, \sigma) \operatorname{sn}(b, \sigma),
\end{aligned}
$$

$b$ and $\sigma$ are defined by formula (13), the function $m(x, t, \varepsilon)$ and $\mathscr{L} u_{0}$ can vanish at finitely many points $(x, t)=\left(x_{k}(\varepsilon), t_{k}(\varepsilon)\right), k=1, \ldots, K, K \in \mathbb{N}$.

Thus, it follows from estimates $(12)$ that the fraction of $\mathscr{L} u_{0}$ and the sum of the absolute values of the derivatives is of order $\rho / \sqrt{\varepsilon}+\sqrt{\rho \varepsilon}$ since exactly this fraction is important and not the function $\mathscr{L} u_{0}$ itself, which can be, generally speaking, large. This exactly corresponds to the relation between the parameters $\rho, \varepsilon$ assumed in Section 1 . We note that the notion of the asymptotic solution we use here differs from the standard definition of a formal asymptotic solution as of an infinite series giving arbitrarily small error term while substituting it into the equation.

In the case, when an asymptotic solution is constructed as a function (and not as a series), even the smallness of the error term arising while substituting this function into the equation can not serve as a good criterion for its suitability since the derivatives can be of the same order. Therefore, we need to compare the error term with something. The most appropriate choice is to choose the absolute values of the terms involved in the equation for comparing.

Proof of Theorem 1. We denote by $\Lambda_{1,2}^{ \pm}$the coefficients of the asymptotics of the function $\Lambda \in$ $C^{\infty}(\mathbb{R}):$

$$
\begin{array}{ll}
\Lambda(s)=a+\frac{\Lambda_{1}^{-}}{s}+\frac{\Lambda_{2}^{-}}{s^{2}}+O\left(\frac{1}{s^{3}}\right), \quad s \rightarrow-\infty, \\
\Lambda(s)=\frac{\Lambda_{1}^{+}}{s}+\frac{\Lambda_{2}^{+}}{s^{2}}+O\left(\frac{1}{s^{3}}\right), \quad s \rightarrow+\infty .
\end{array}
$$

Employing (11), Lemma 1 and the asymptotics of the function $\Lambda(s)$ as $s \rightarrow \pm \infty$, we obtain

$$
u_{0}=G(x, t, \xi, \varepsilon)+\frac{2 \Lambda_{1}^{+} \rho}{x+a t}-\frac{\Lambda_{1}^{-} \rho}{x-2 a t / 3}+O(\sqrt{\rho})
$$

where

$$
G=a\left[2 \operatorname{dn}^{2}(b, \sigma)+\sigma^{2}-1\right], \quad b=\frac{a^{3 / 2}}{\sqrt{6 \varepsilon}}\left(\frac{x}{a}-\frac{t\left(1+\sigma^{2}\right)}{3}\right), \quad \sigma=\sigma\left(\frac{x}{a t}\right) .
$$

Differentiating (11) w.r.t. $t, x$ and applying Lemma 1 as above, we obtain

$$
\begin{aligned}
& \frac{\partial u_{0}}{\partial t}=G_{t}+O\left(\frac{\rho}{\varepsilon}+\sqrt{\rho}\right), \\
& \frac{\partial u_{0}}{\partial x}=G_{x}+O\left(\frac{\rho}{\varepsilon}+\sqrt{\rho}\right),
\end{aligned}
$$




$$
\frac{\partial^{3} u_{0}}{\partial x^{3}}=G_{x x x}+O\left(\frac{\rho}{\varepsilon} \frac{1}{\varepsilon}+\sqrt{\rho}\right) .
$$

We substitute the linear increment $\sigma_{0}+\sigma^{\prime}(\tilde{\zeta})\left(\zeta-\zeta_{0}\right)$ into $G$ instead of $\sigma$, where $\sigma_{0}=\sigma\left(\zeta_{0}\right)$ and differentiate w.r.t. $t, x$. For $\zeta=\frac{x}{a t} \rightarrow \zeta_{0}=\frac{x_{0}}{a t_{0}}$ we have

$$
\begin{aligned}
& G_{t}=\frac{2 \sqrt{6} a^{5 / 2} \sigma_{0}^{2}\left(1+\sigma_{0}^{2}\right) \operatorname{dn}\left(b_{0}, \sigma_{0}\right) \operatorname{cn}\left(b_{0}, \sigma_{0}\right) \operatorname{sn}\left(b_{0}, \sigma_{0}\right)}{9 \sqrt{\varepsilon}}+O\left(\frac{t\left(\zeta-\zeta_{0}\right)}{\varepsilon}\right), \\
& G_{x}=-\frac{2 \sqrt{6} a^{3 / 2} \sigma^{2} \operatorname{dn}\left(b_{0}, \sigma_{0}\right) \operatorname{cn}\left(b_{0}, \sigma_{0}\right) \operatorname{sn}\left(b_{0}, \sigma_{0}\right)}{3 \sqrt{\varepsilon}}+O\left(\frac{t\left(\zeta-\zeta_{0}\right)}{\varepsilon}\right), \\
& G_{x x x}=\frac{4 \sqrt{6} a^{5 / 2} \sigma^{2} \operatorname{dn}\left(b_{0}, \sigma_{0}\right) \operatorname{cn}\left(b_{0}, \sigma_{0}\right) \operatorname{sn}\left(b_{0}, \sigma_{0}\right)\left(\sigma_{0}^{2}+1-3 \sigma_{0}^{2} \operatorname{sn}^{2}\left(b_{0}, \sigma_{0}\right)\right)}{9 \varepsilon^{3 / 2}}+O\left(\frac{t\left(\zeta-\zeta_{0}\right)}{\varepsilon^{2}}\right),
\end{aligned}
$$

uniformly in all $\zeta_{0}, \zeta \in(-1+\mu, 2 / 3-\mu)$ for $\mu>0$, where

$$
b_{0}=\frac{a^{3 / 2}}{\sqrt{6 \varepsilon}}\left(\frac{x_{0}}{a}-\frac{t_{0}\left(1+\sigma_{0}^{2}\right)}{3}\right), \quad \sigma_{0}=\sigma\left(\frac{x_{0}}{a t_{0}}\right)
$$

and $\operatorname{cn}(b, \sigma), \operatorname{sn}(b, \sigma)$, as well as $\operatorname{dn}(b, \sigma)$ are standard Jacobi elliptic functions. This implies the second estimate in (12). The function $m(x, t, \varepsilon)$ is equal to the function $\operatorname{dn}(b, \sigma) \operatorname{cn}(b, \sigma) \operatorname{sn}(b, \sigma)$ up to some multiplicative constant, which oscillates w.r.t. $x$ and $t$ for a frozen $\sigma$ and it vanishes at some points:

$$
m(x, t, \varepsilon)=\frac{4 \sqrt{6} a^{5 / 2} \sigma^{2}}{3} \operatorname{dn}^{3}(b, \sigma) \operatorname{cn}(b, \sigma) \operatorname{sn}(b, \sigma)
$$

Moreover,

$$
G_{t}+G G_{x}+\varepsilon G_{x x x}=O\left(\frac{t\left(\zeta-\zeta_{0}\right)}{\varepsilon}\right)
$$

uniformly in all $\zeta_{0}, \zeta \in(-1+\mu, 2 / 3-\mu)$ for $\mu>0$. For each ray $\zeta=\frac{x}{a t}$ we can choose arbitrarily close value $\zeta_{0}$. Therefore,

$$
\frac{\partial u_{0}}{\partial t}+u_{0} \frac{\partial u_{0}}{\partial x}+\varepsilon \frac{\partial^{3} u_{0}}{\partial x^{3}}=G_{t}+G G_{x}+\varepsilon G_{x x x}+O\left(\frac{\rho}{\varepsilon}+\sqrt{\rho}\right)
$$

and we obtain the first estimate in 12 . The proof is complete.

\section{NUMERICS}

In the present section we give the graphs of the asymptotic solutions of the considered problem. We obtain them the system of symbolic calculates Maple. The function $\sigma(y)(10)$ is approximated by corresponding Taylor series in four domains. To construct the integrand in (11), we employ cubic splines with the nodes at the extremum points and approximately with 200 segments. Since the derivatives of the Gurevich-Pitaevskii solution has singularities at the wave fronts, we do not include in Figures 4 , 5 the parts of the graphs containing these singularities. 
1. Initial function $\Lambda=\frac{\pi}{2}-\arctan \frac{x}{\rho}$.

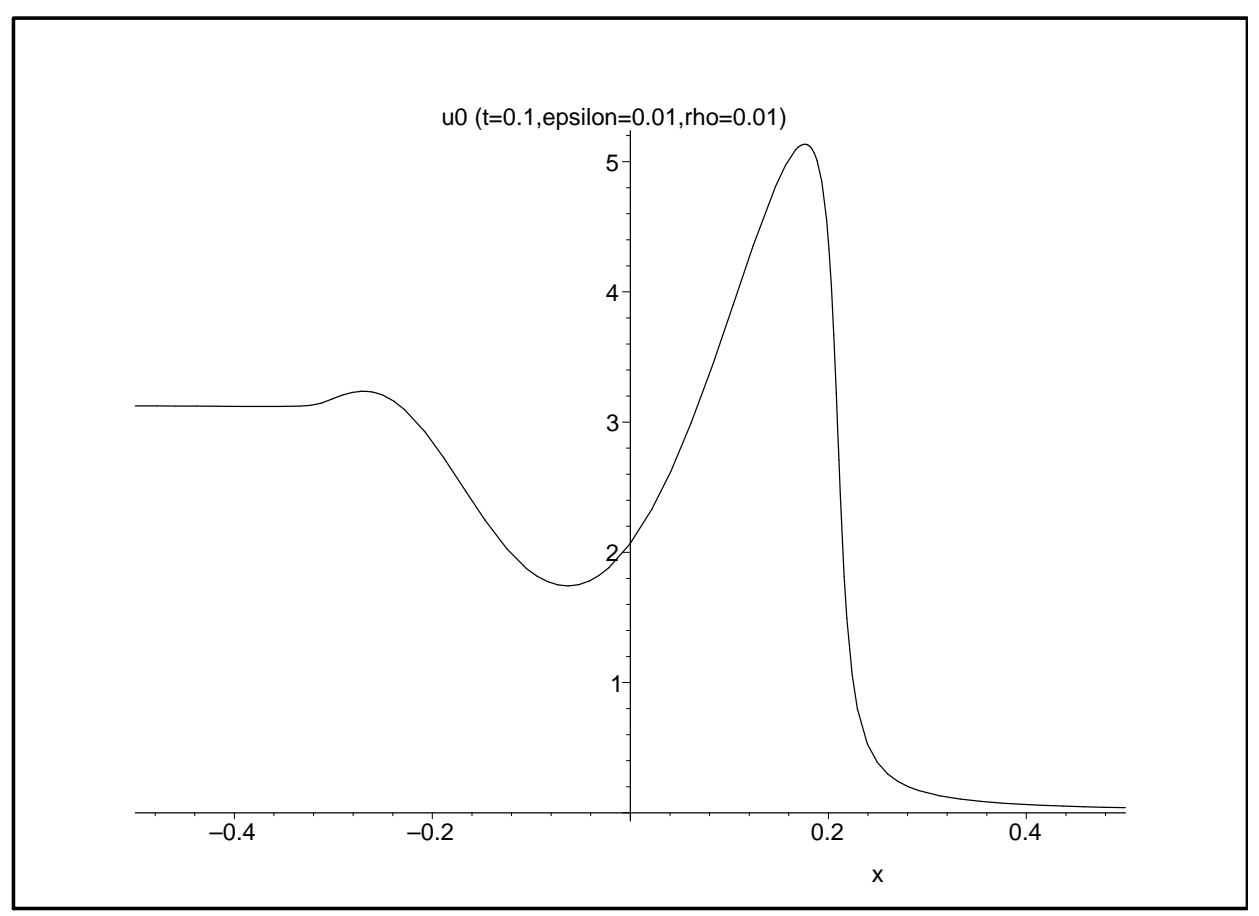

FiguRE 1. $t=0.1$

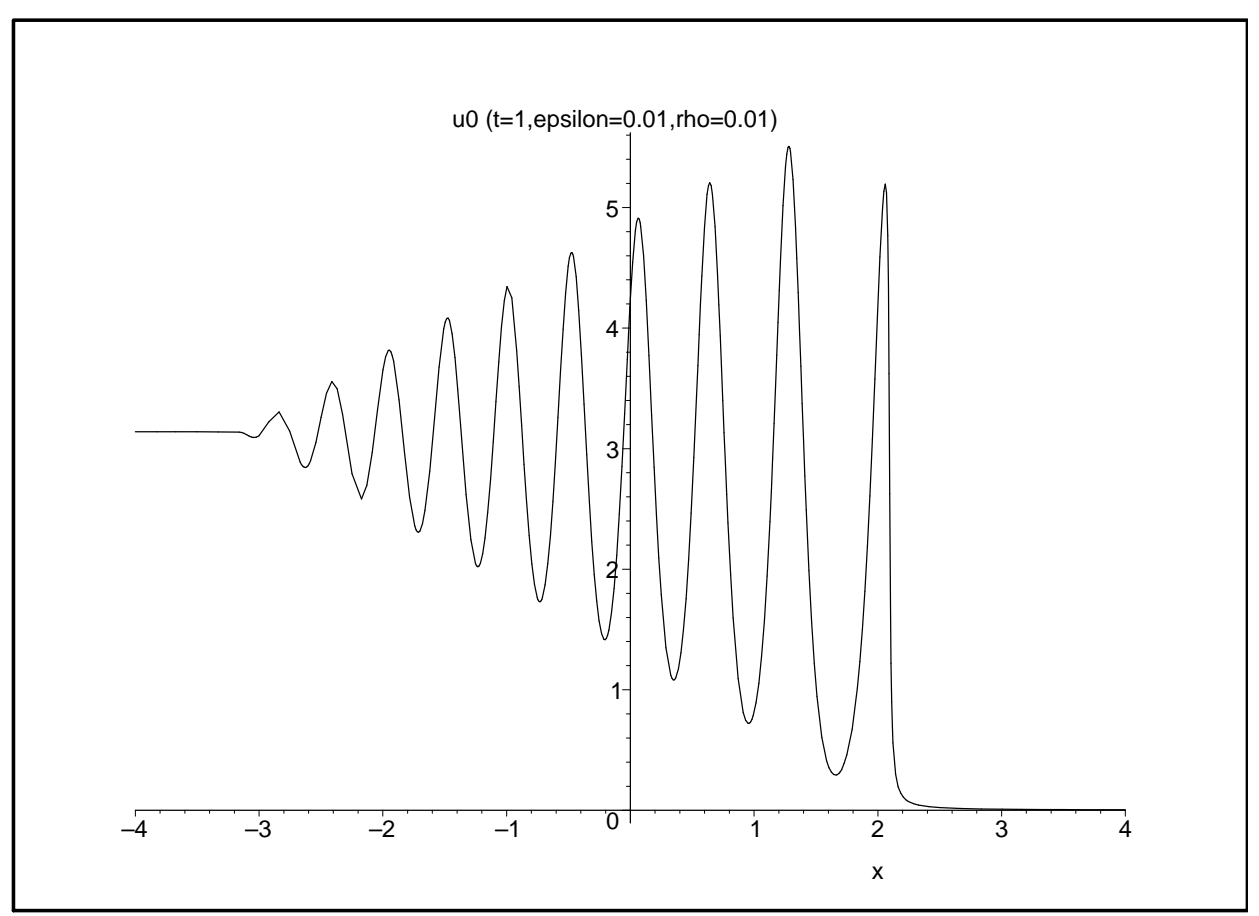

FigURE 2. $t=1$ 


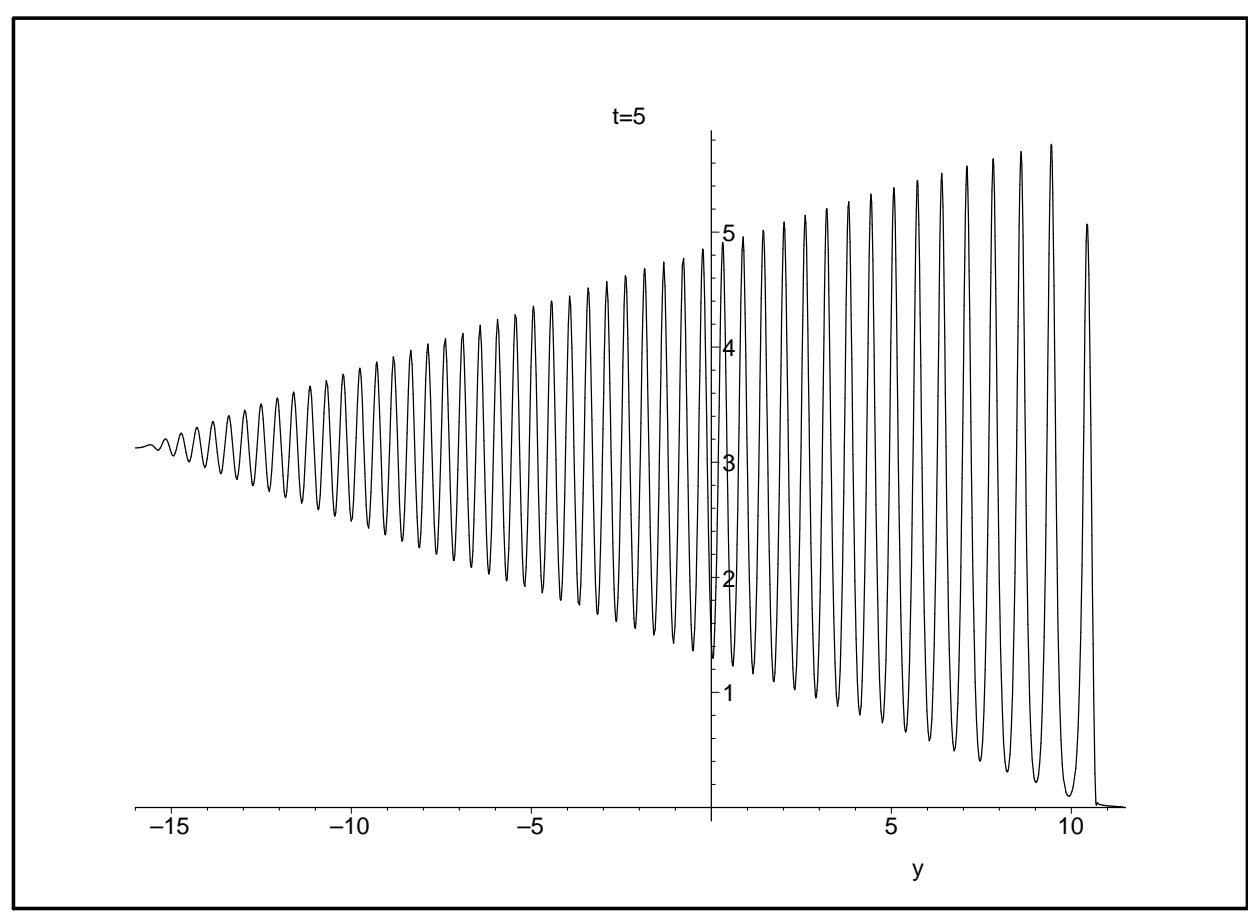

FIGURE 3. $t=5$

2. Numerical estimate of the value of KdV operator.

For the approximate solution $u_{0}$ we denote

$$
m_{1}(x)=\left|\frac{\partial u_{0}}{\partial t}+u_{0} \frac{\partial u_{0}}{\partial x}+\varepsilon \frac{\partial^{3} u_{0}}{\partial x^{3}}\right|, \quad m_{2}(x)=\left|\frac{\partial u_{0}}{\partial t}\right|+\left|u_{0} \frac{\partial u_{0}}{\partial x}\right|+\varepsilon\left|\frac{\partial^{3} u_{0}}{\partial x^{3}}\right| .
$$

As we see in Figure 4, 5, the quantity $m_{1}$ turns out to be much smaller than quantity $m_{2}$. We stress once again that the smallness of the fraction $m_{1}$ and $m_{2}$ is important, while the quantity $m_{1}$ is not necessarily small.

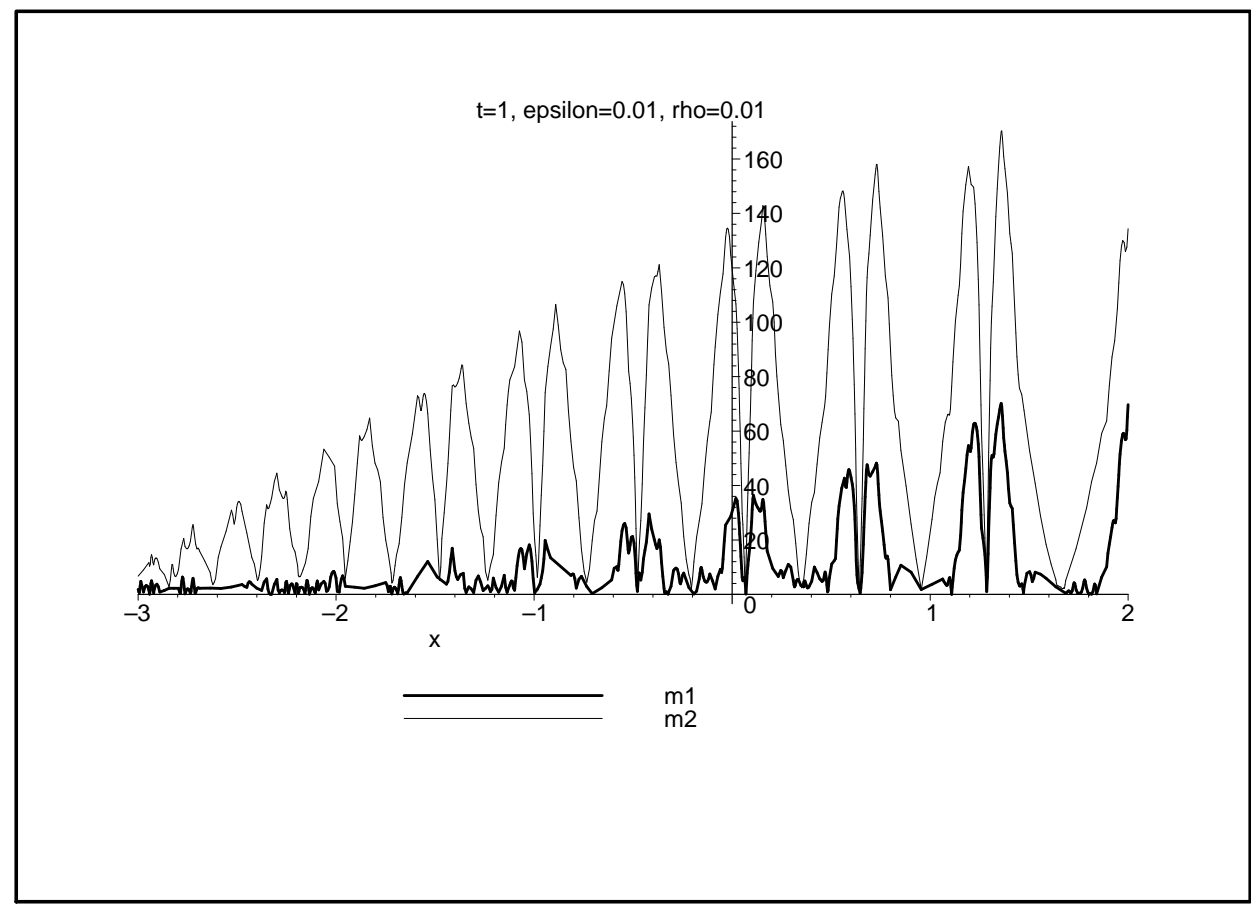

FigURE 4. $t=1$ 


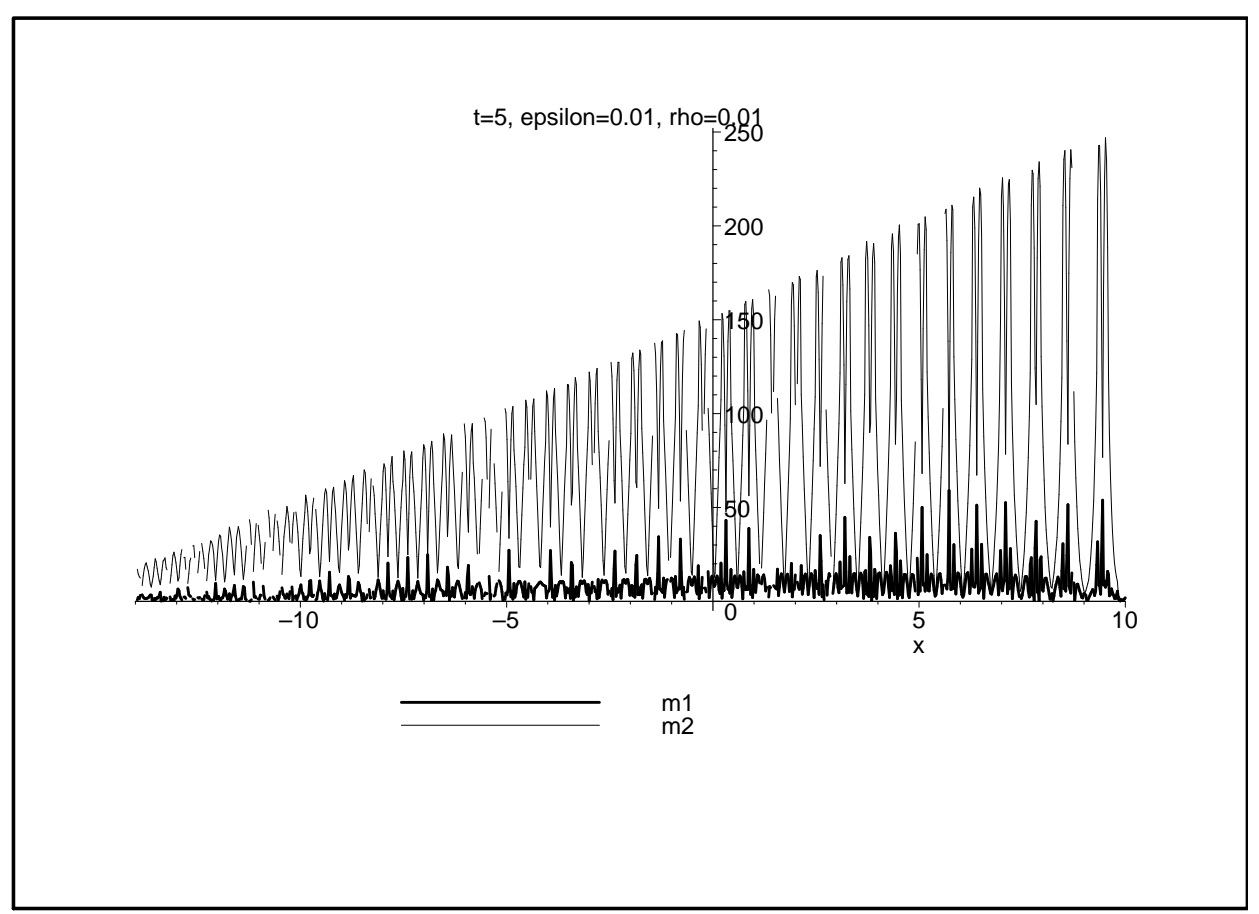

FIGURE $5 . t=5$

3. Initial function $\Lambda_{1}=\left(\frac{\pi}{2}-\arctan \frac{x}{\rho}\right)\left(1-3 e^{-(x / \rho)^{2}}\right)$.

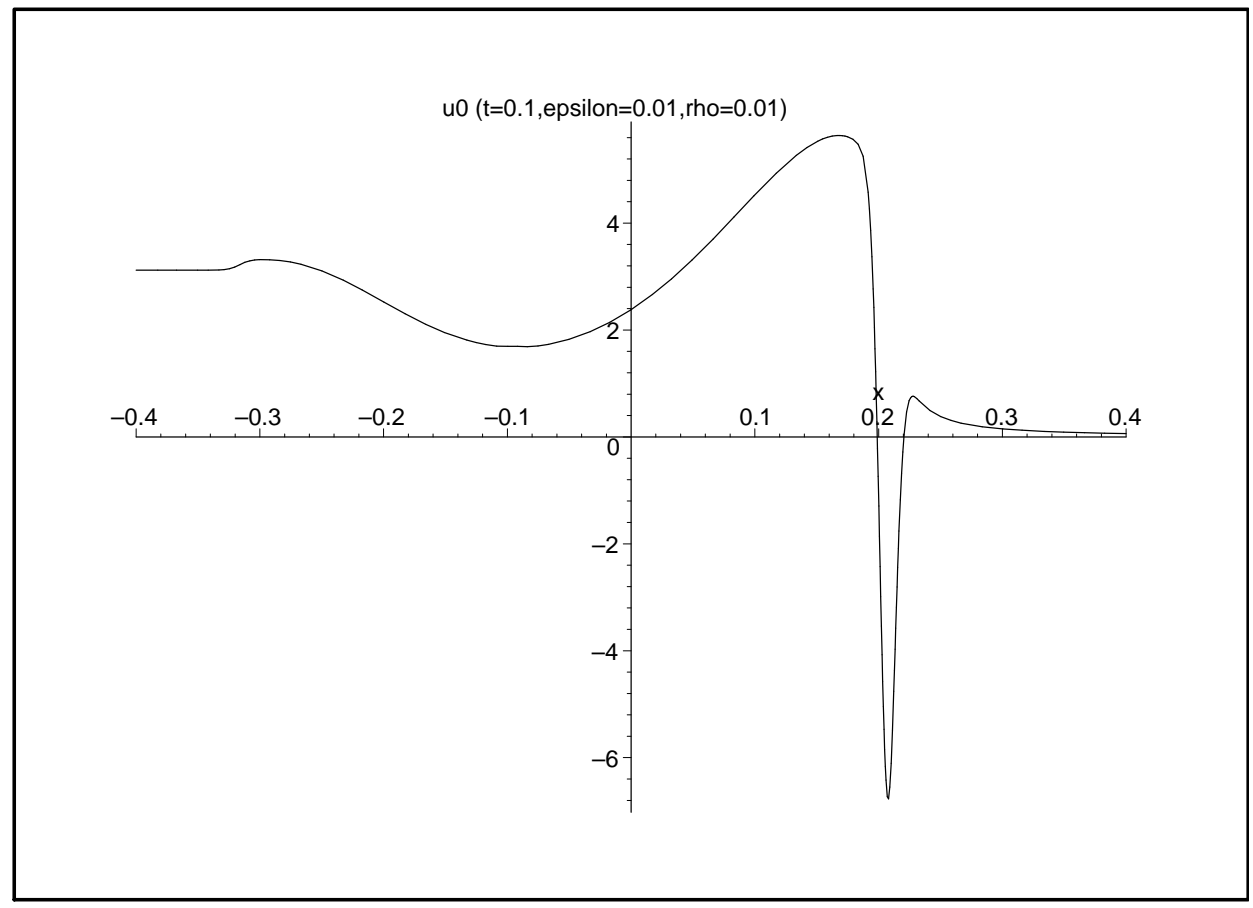

Figure $6 . \Lambda_{1}: t=0.1$ 


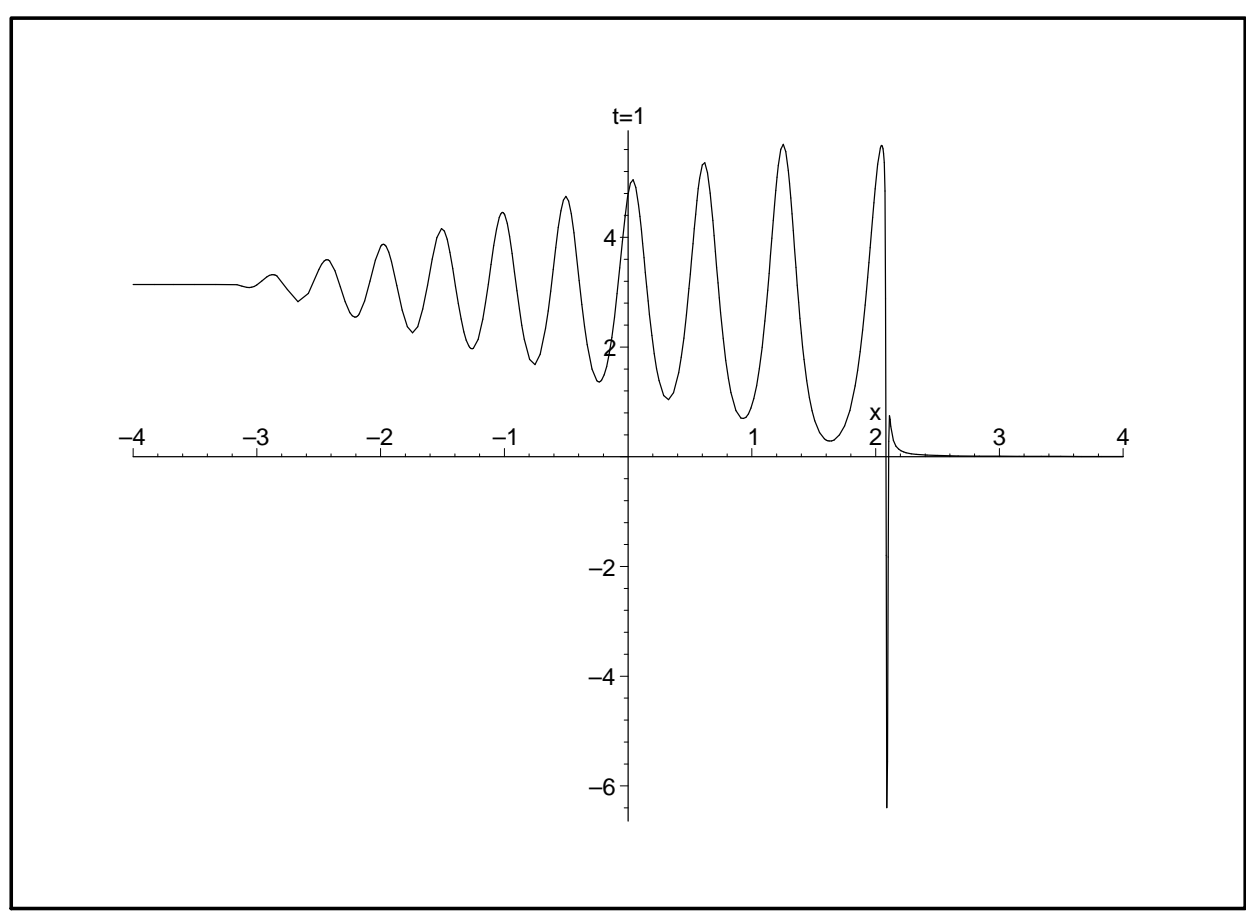

Figure $7 . \Lambda_{1}: t=1$

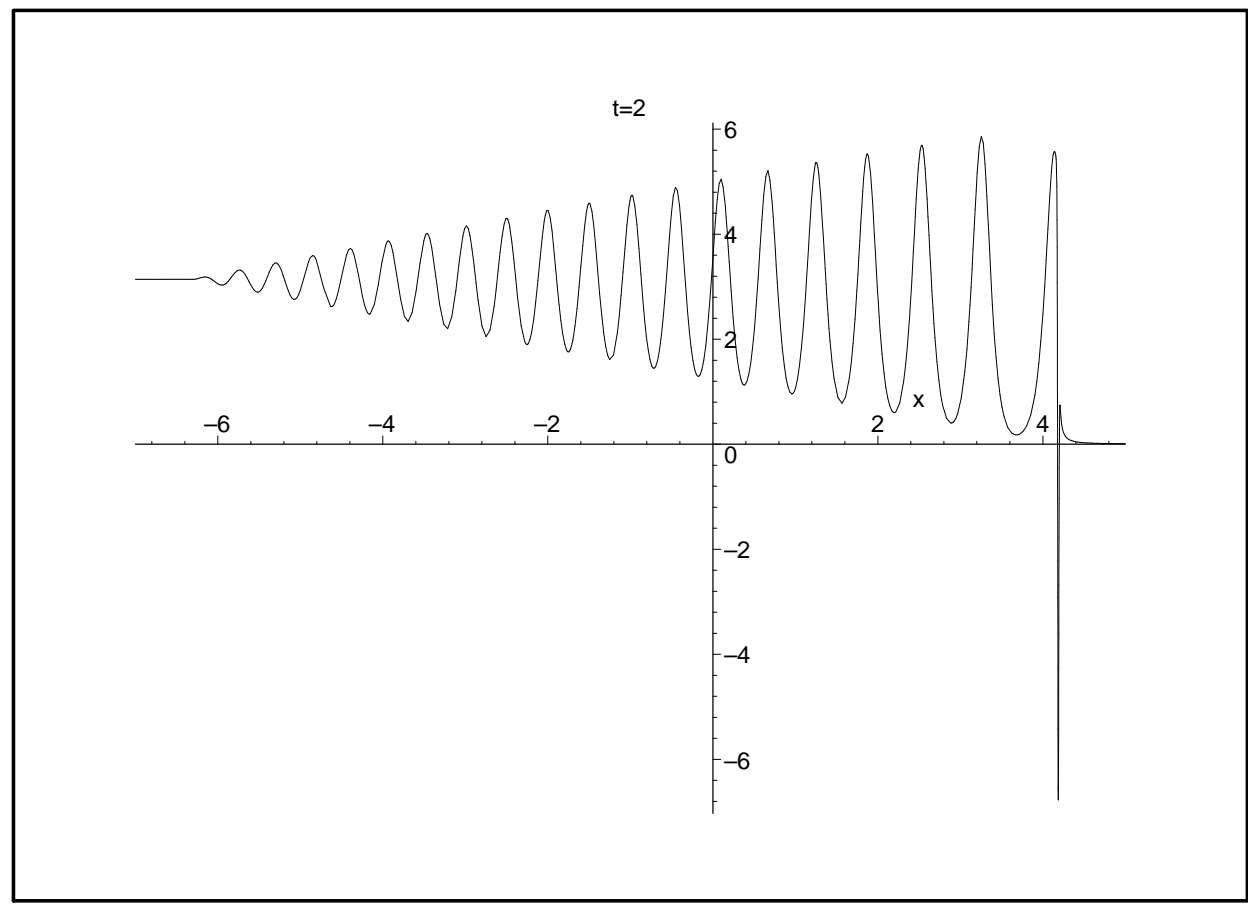

Figure $8 . \Lambda_{1}: t=2$ 
4. Initial function $\Lambda_{2}=\left(\frac{\pi}{2}-\arctan \frac{x}{\rho}\right)\left(1+3 e^{-(x / \rho)^{2}}\right)$.

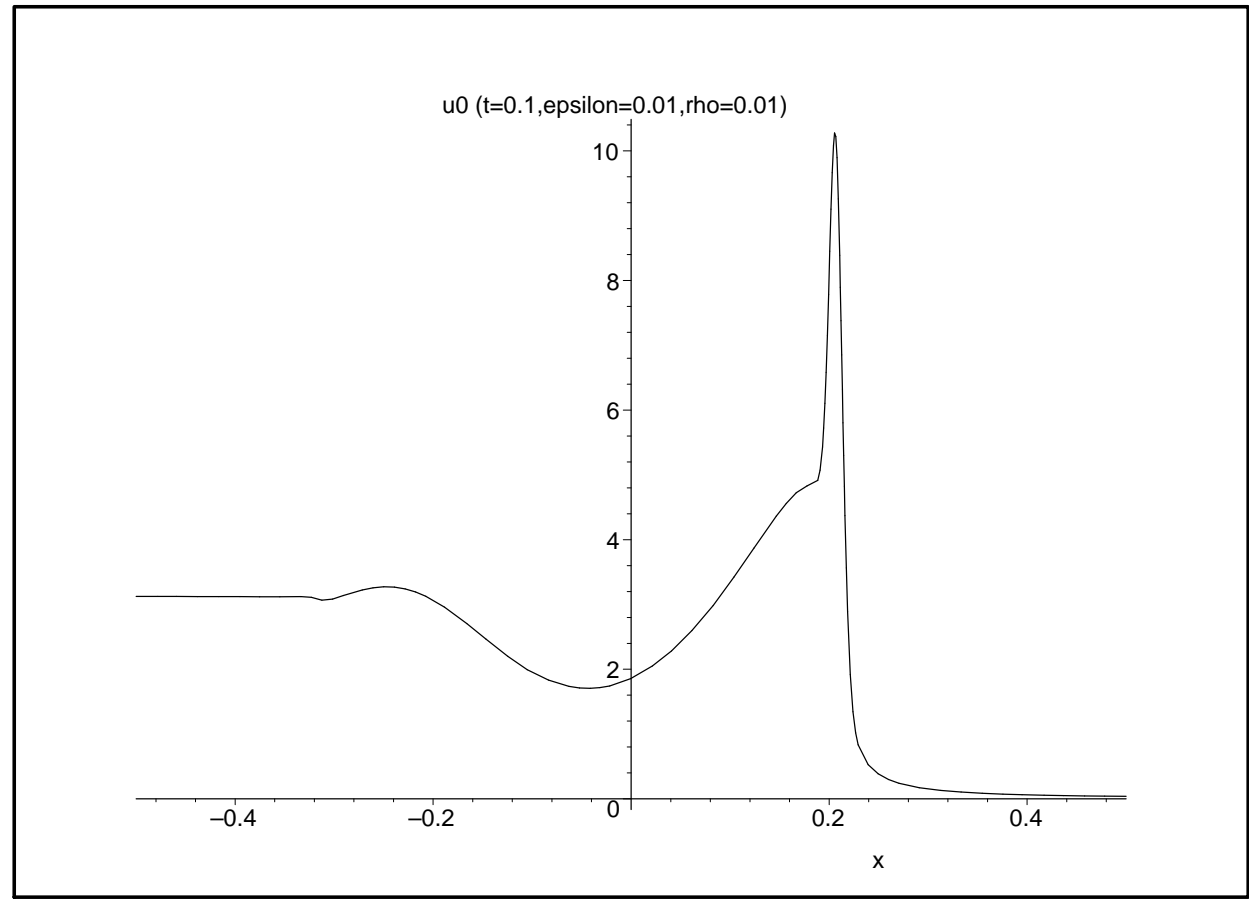

Figure $9 . \quad \Lambda_{2}: t=0.1$

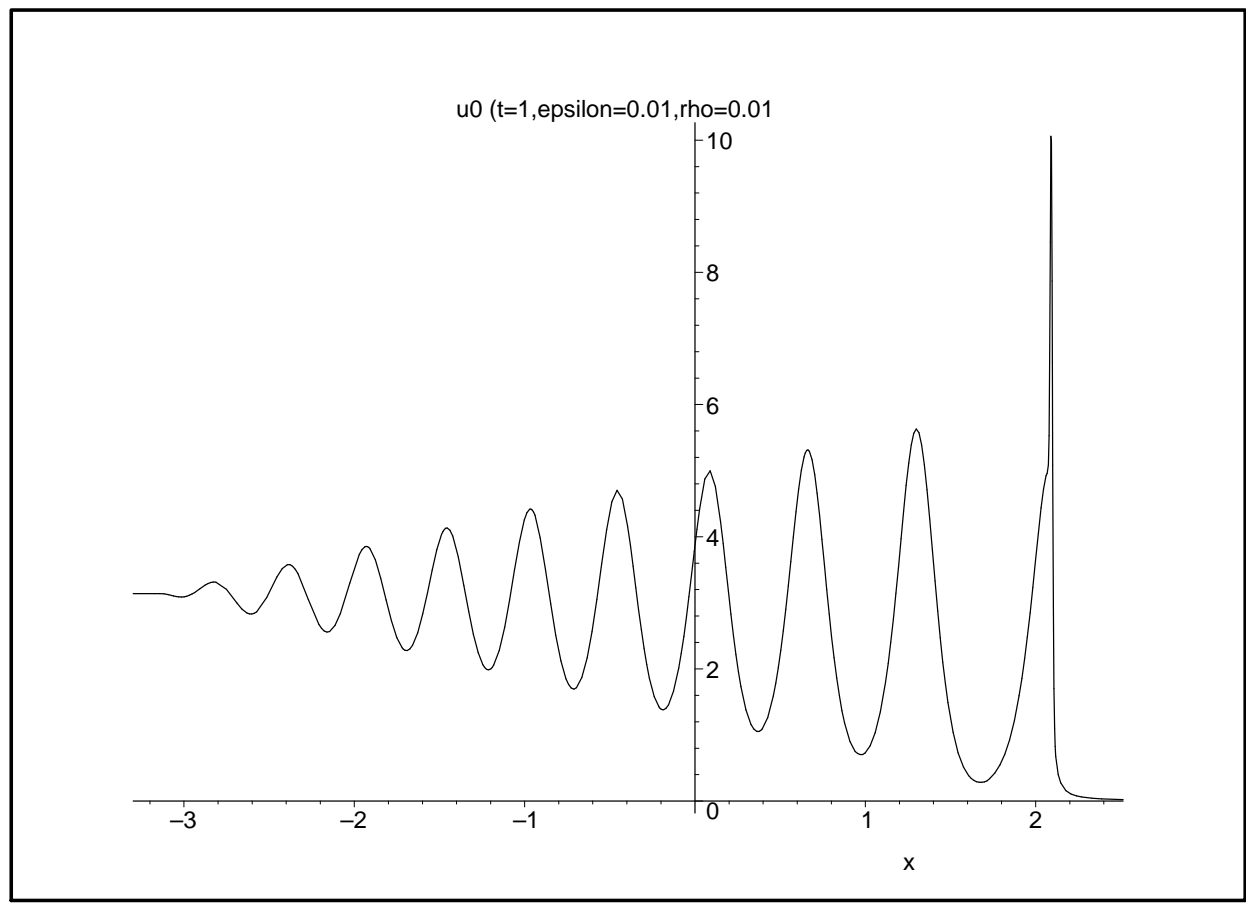

FiguRE $10 . \Lambda_{2}: t=1$ 


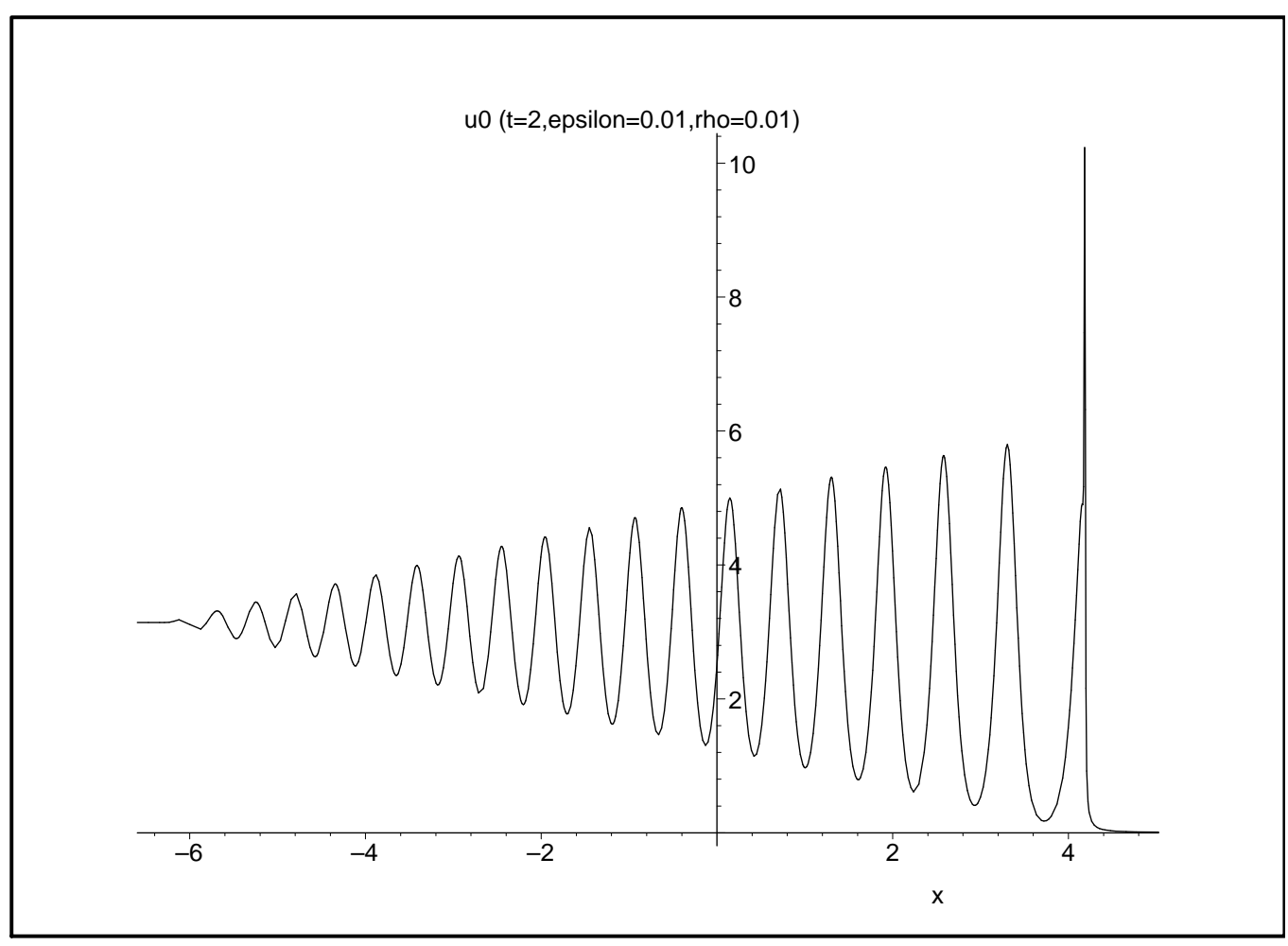

FiguRE $11 . \Lambda_{2}: t=2$

The authors thank B.I. Suleimanov for discussions.

\section{BIBLIOGRAPHY}

1. A.V. Gurevich, L.P. Pitayevskii. Nonstationary structure of a collisionless shock wave // Zhurn. Eksper. Teor. Fiz. 65:2, 590-604 (1974). [J. Exp. Theor. Phys. 38:2, 291-297 (1974).]

2. A.V. Gurevich, A.L. Krylov, G.A. El'. Breaking of a Riemann wave in dispersive hydrodynamics // Pis'ma v ZhETF. 54:2, 104-109 (1991). [JETP Lett. 54:2, 102-107 (1991).]

3. A.L. Krylov, V.V. Khodorovskii, G.A. El'. Evolution of a nonmonotonic perturbation in Kortewegde Vries hydrodynamics // Pis'ma v ZhETF. 56:6, 325-429 (1992). [JETP Lett. 56:6, 323-327 (1992).]

4. N. G. Mazur. Quasiclassical asymptotics of the inverse scattering solutions of the KdV equation and the solution of Whitham's modulation equations // Teor. Matem. Fiz. 106:1, 44-61 (1996). [Theor. Math. Phys. 106:1, 35-49 (1996).]

5. E.Ya. Khruslov. Asymptotics of the solution of the Cauchy problem for the Korteweglide Vries equation with initial data of step type // Matem. Sborn. 99:2, 261-281 (1976). [Math. USSR-Sb. 28:2, 229-248 (1976).]

6. A. Cohen. Solutions of the Korteweg-de Vries equation with steplike initial profile// Comm. Part. Diff. Equat. 9:8, 751-806 (1984).

7. S. Venakides. Long time asymptotics of the Korteweg-de Vries equation // Trans. Amer. Math. Soc. 293:1, 411-419 (1986).

8. B.I. Suleimanov. Solution of the Korteweg-de Vries equation which arises near the breaking point in problems with a slight dispersion // Pis'ma v ZhETF. 58:11, 906-910 (1993). [JETP Lett. 58:11, 849-854 (1993).

9. T. Kappeler. Solutions of the Korteweg-de Vries equation with steplike initial data // J. Diff. Equats. 63:3, 306-311 (1986).

10. I.N. Bondareva. The Korteweg-de Vries equation in classes of increasing functions with prescribed asymptotics as $|x| \rightarrow \infty / /$ Matem. Sborn. 122(164):2(10), 131-141 (1983). [Math. USSR-Sb. 50:1, 125-135 (1985).] 
11. S.V. Zakharov. Renormalization in the Cauchy problem for the Korteweg-de Vries equation // Teor. Matem. Fiz. 175:2, 173-177 (2013). [Theor. Math. Phys. 175:2, 592-595 (2013).]

12. E.V. Teodorovich. Renormalization group method in the problems of mechanics // Prikl. Mat. Mekh. 68:2, 335-367 (2004). [J. Appl. Math. Mech. 68:2, 299-326 (2004).]

13. A.M. Il'in. Matching of asymptotic expansions of solutions of boundary value problems // Nauka, Moscow (1989). [Transl. Math. Monog. 102. Amer. Math. Soc., Providence, RI (1992).]

14. S.V. Zakharov. The Cauchy problem for a quasilinear parabolic equation with two small parameters // Dokl. Akad. Nauk. 422:6, 733-734 (2008). [Dokl. Math. 78:2, 769-770 (2008).]

15. S.V. Zakharov. The Cauchy problem for a quasilinear parabolic equation with a large initial gradient and low viscosity // Zhurn. Vychisl. Matem. Matem. Fiz. 50:4, 699-706 (2010). [Comp. Math. Math. Phys. 50:4, 665-672 (2010).]

Zakharov Sergey Viktorovich

N.N. Krasovskii Institute of Mathematics and Mechanics,

S. Kovalevskaya str., 16

620990, Ekaterinburg, Russia

E-mail: svz@imm.uran.ru

El'bert Alexander Evgen'evich

N.N. Krasovskii Institute of Mathematics and Mechanics,

S. Kovalevskaya str., 16

620990, Ekaterinburg, Russia

E-mail: aee@imm.uran.ru 